\title{
Photothermal Investigation of Te-Doped Bulk Gasb: Correlation between Non-Radiative Lifetime and Thermal Diffusivity
}

\author{
Faycel Saadallah", Sameh Abroug, Noureddine Yacoubi \\ Photothermal Laboratory of Nabeul, Merazka Nabeul, 8050, Tunisia
}

\begin{abstract}
Photothermal deflection PTD is used in order to reveal the influence of Te doping on non radiative carrier lifetime for GaSb bulk sample when illuminated by a modulated and monochromatic light beam. Theoretical simulations are obtained from an adapted theoretical model, based on the resolution of both heat and carrier diffusion equations. Auger recombination coefficient obtained from the linear relation between lifetime and the inverse of squared concentration is in agreement with literature. Moreover, it is found that low n-doping reduces the surface recombination velocity $\mathrm{S}$ because of dangling bonds neutralisation at the surface. However for higher doping concentration, $\mathrm{S}$ is enhanced by Te doping.
\end{abstract}

Keywords Photothermal Deflection, Lifetime, Thermal Diffusivity, Doping

\section{Introduction}

Carrier transport mechanisms in semiconductors are widely investigated by many techniques[1-5] such as time resolved photoconductivity, photoluminescence and photoacoustic processes. Of the several techniques available for semiconductor characterization, photothermal deflection PTD [5-9] is one of the most widely used. In this case, the sample is usually irradiated by a modulated light beam. The absorbed energy is converted into heat that diffuses in the surrounding fluid causing the deflection of a probe beam skimming the sample surface. The measured deflection also called photothermal signal provides us, besides thermal and optical parameters, with carrier transport properties such as lifetime of photo-induced free carriers. Indeed, generation and recombination of minority carriers occur in a thin layer near the irradiated surface and thus has a significant contribution to the photothermal signal.

In this paper, we present PTD measurement of minority carrier lifetime in doped GaSb. As the measured photothermal signal is proportional to the probe beam deflection, we should calculate the sample surface temperature since it is supposed to be proportional to this deflection. For this purpose, we propose a theoretical model based on the resolution of both heat and carrier diffusion equations. Then we fit the amplitude and phase of the theoretical deflection to those of the measured signal with bulk lifetime and surface

* Corresponding author:

Faycel1@yahoo.fr (Faycel Saadallah)

Published online at http://journal.sapub.org/ajcmp

Copyright (C 2012 Scientific \& Academic Publishing. All Rights Reserved recombination velocity $S$ as adjustable parameters. The heat generated is related to the non radiative processes, and therefore the parameters that can be determined through this procedure are and $\mathrm{S}$. Non radiative recombination can occur in several ways. In high quality GaSb samples, the main contribution to non-radiative carrier lifetime is most probably, the band to band recombination for which Auger process can be the dominant mechanism.

\section{Theoretical Model}

\subsection{Principle of Photothermal Deflection}

The semiconductor sample is heated by a uniform monochromatic Light beam, modulated at the frequency F, which strikes normally at the sample surface. When the surface is uniformly illuminated, thermal wave generated by the optical absorption diffuses only in the beam direction through the sample and the surrounding fluid. The temperature variations in the fluid (air) induces a refractive index gradient which causes the deflection of a laser probe beam parallel to the sample surface passing through the fluid. This deflection could be related to thermal and carrier transport properties of the sample. The complex expression of the deflection $\Phi$ is given by[9]:

$$
\Phi=\frac{L_{s}}{n} \frac{d n}{d T} \sigma_{f} \exp \left(-\sigma_{f} x_{0}\right) T_{0}
$$

where $\mathrm{n}$ is the fluid index, $\mathrm{L}_{\mathrm{s}}$ is the sample width in the laser beam direction, $\mathrm{x}_{0}$ the distance between the probe beam axis and the sample surface, $\sigma_{f}=\frac{1+i}{\mu_{f}}$ is related to thermal diffusion length $\mu_{f}=\sqrt{\frac{D_{f}}{\pi F}}$ in the fluid, and $\mathrm{T}_{0}$ is the complex 
temperature rise at the sample surface written as:

$$
T_{0}=\left|T_{0}\right| \exp \left(\begin{array}{ll}
i & \theta
\end{array}\right)
$$

Finally, we find that deflection and T0 amplitudes are proportional throw :

$$
|\Phi|=\frac{L_{s}}{n} \frac{d n}{d T} \frac{1}{\mu_{f}} \quad \exp \left(-\frac{x_{0}}{\mu_{f}}\right) \quad\left|T_{0}\right|
$$

and their phases have the same variations:

$$
\arg (\Phi)=-\frac{x_{0}}{\mu_{f}}+\theta+\frac{\pi}{4}=\text { constan } t+\theta
$$

In order to determine the amplitude and phase of the theoretical signal we should calculate the surface temperature $\mathrm{T} 0$, this will be the purpose of the next paragraph.

\subsection{Determination of the Surface Temperature $T_{0}$}

In order to calculate the temperature at the sample surface, we should solve the heat and carrier diffusion equations. This method has been followed in Photoacoustic investigations [10-12]. The same equations are used in PTD[12] with the assumption of optically thin sample, which is not the case in this work where bulk GaSb have been studied.

In our case, as the sample (figure 1) is uniformly heated, only diffusion in the heating beam direction is considered. This assumption is satisfied if the pump beam dimensions are higher than thermal diffusion length.

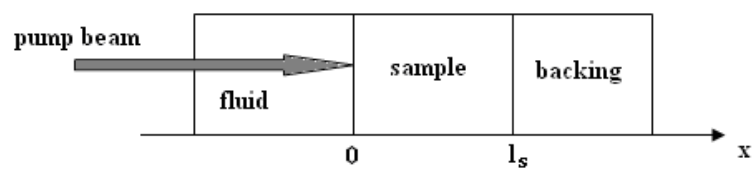

Figure 1. Schema of the sample with a backing and the deflecting medium

For semiconductor samples, in addition to thermalization process, the recombination of photo-excited electron-hole pairs contributes to the heat transfer. Therefore, diffusion of these carriers should be considered throw the carrier diffusion equation in the direction $\mathrm{x}$ of pump beam:

$$
\frac{\partial \tilde{P}}{\partial t}=D \frac{\partial^{2} \tilde{P}}{\partial x^{2}}-\frac{\tilde{P}}{\tau}+H_{0} e^{-\alpha x} e^{i \omega t}
$$

$\mathrm{P}, \mathrm{D}$, and $\mathrm{R}$ are respectively, density of photo-generated carriers, carrier diffusion coefficient, absorption coefficient and reflectivity of the sample, $\omega=2 \pi F$ and $H_{0}=\frac{\alpha I_{0}(1-R)}{2 E}$ is the generation term which depends on the intensity $I_{0}$ of heating beam, its energy $\mathrm{E}$ and the sample reflectivity $\mathrm{R}$.

In the other hand, the heat flow is governed by the heat conduction equation in the different media:

$$
\begin{gathered}
\frac{\partial^{2} \tilde{T}_{s}}{\partial x^{2}}-\sigma_{s}^{2} \tilde{T}_{s}=-\frac{E_{g} \tilde{P}}{\tau k_{s}}+H_{0} \frac{\left(E-E_{g}\right)}{k_{s}} e^{-\alpha x} \\
\frac{\partial^{2} \tilde{T}_{f}}{\partial x^{2}}-\sigma_{f}^{2} \tilde{T}_{f}=0 \\
\frac{\partial^{2} \tilde{T}_{b}}{\partial x^{2}}-\sigma_{b}^{2} \tilde{T}_{b}=0
\end{gathered}
$$

$\tilde{T}_{j}=T_{j} e^{i \omega t}$ is the complex temperature in the medium $\mathrm{j}$ $\left(\mathrm{j}=\mathrm{f}, \mathrm{s}\right.$ or $\mathrm{b}$ ) and $\widetilde{\mathrm{P}}=\mathrm{Pe}^{\mathrm{i} \omega \mathrm{t}}$ is the complex excess photo-carrier density.

$\sigma_{j}=\frac{1+i}{\mu_{j}}$ depends on thermal diffusion length $\mu_{j}=\sqrt{\frac{D_{j}}{\pi F}} \mathrm{D}_{\mathrm{j}}$ and $\mathrm{k}_{\mathrm{j}}$ are respectively thermal diffusivity and conductivity of the medium $\mathrm{j}$.

Equation 6 show that the absorbed light energy is converted into heat throw two processes: thermalization and bulk recombination.

The general solutions of the above equations are:

$$
\begin{aligned}
& T_{s}=A_{1} e^{-\sigma_{s} x}+B_{1} e^{\sigma_{s} x}+C_{1} e^{-q_{p} x}+D_{1} e^{-\alpha x} \\
& T_{f}=A_{f} e^{\sigma_{f} x} \\
& T_{b}=A_{b} e^{-\sigma_{b}\left(x-l_{s}\right)}
\end{aligned}
$$

The particular solutions of equation 2 are $\mathrm{C} 1$ and $\mathrm{D} 1$ given by :

$$
\begin{aligned}
& C_{1}=\frac{-E_{g} H_{0}}{\tau k_{s} D\left(\alpha^{2}-q_{p}^{2}\right)\left(q_{p}^{2}-\sigma_{s}^{2}\right)}\left(\frac{D \alpha+S}{D q_{p}+S}\right) \\
& D_{1}=\frac{-E_{g} H_{0}}{\tau k_{s} D\left(\alpha^{2}-q_{p}^{2}\right)\left(\alpha^{2}-\sigma_{s}^{2}\right)}
\end{aligned}
$$

Then, the continuity equations of temperature and heat flux are used to determine the other parameters (A1, B1 and Af):

$$
\begin{gathered}
T_{s}(x=0)=T_{f}(x=0) \\
T_{s}\left(x=l_{s}\right)=T_{b}\left(x=l_{s}\right) \\
-k_{s} \frac{\partial T_{s}}{\partial x}\left(x=l_{s}\right)=-k_{b} \frac{\partial T_{b}}{\partial x}\left(x=l_{s}\right) \\
-k_{s} \frac{\partial T_{s}}{\partial x}(x=0)=-k_{f} \frac{\partial T_{f}}{\partial x}(x=0)+S E_{g} P(x=0)
\end{gathered}
$$

Here Eg is the bandgap energy.

From these conditions we get:

$$
\begin{gathered}
A_{1}=\frac{A_{01}}{\left(-c_{s b}+1\right)\left(c_{s f}-1\right) e^{-\sigma_{s} l_{s}}+\left(-c_{s b}+1\right)\left(c_{s f}+1\right) e^{\sigma_{s} l_{s}}} \\
A_{01}=\left\{C_{1}\left[e^{-q_{p} l_{s}}\left(t_{p b}-1\right)\left(c_{s f}-1\right)-e^{-\sigma_{s} l_{s}}\left(t_{p f}+1\right)\left(c_{s b}+1\right)\right]+\right. \\
\left.D_{1}\left[e^{-\alpha l_{s}}\left(r_{s b}-1\right)\left(c_{s f}-1\right)-e^{-\sigma_{s} l_{s}}\left(r_{s f}+1\right)\left(c_{s b}+1\right)\right]+\frac{W_{0} e^{\sigma_{s} l_{s}}\left(c_{s b}+1\right)}{k_{f} \sigma_{f}}\right\} \\
B_{1}=\frac{1}{\left(c_{s f}-1\right)}\left[A_{1}\left(c_{s f}+1\right)+C_{1}\left(t_{s p}+1\right)+D_{1}\left(r_{s f}-1\right)-\frac{W_{0}}{k_{f} \sigma_{f}}\right]
\end{gathered}
$$

where:

$$
W_{0}=\frac{\left(E-E_{g}\right) H_{0}}{\alpha}+\frac{S E_{g} H_{0}}{\left(\alpha+q_{p}\right)\left(D q_{p}+S\right)}
$$

Finally, we obtain the surface temperature given by:

$$
\mathrm{T} 0=\mathrm{Af}=\mathrm{A} 1+\mathrm{B} 1+\mathrm{C} 1+\mathrm{D} 1
$$

\section{Experimental Setup}

The experimental device[8] is shown in fig.2. Monochromatic light beam is modulated with a mechanical 
chopper at a frequency $\mathrm{F}$ and focused on the sample surface. The absorption of the optical heating beam by the sample generates a thermal wave that propagates in the sample and in the deflecting fluid (air), leading to the deflection of a probe laser beam $(\mathrm{He}-\mathrm{Ne})$ skimming the sample surface. This deflection is detected with a position photodetector linked to a lock in amplifier giving us the amplitude and phase of the measured photothermal signal. The curves are drawn while varying the modulation frequency $\mathrm{F}$ in the range of 10-400 Hz. Indeed, some works[13] show that we can determine the transport properties and S) of bulk semiconductors in this frequency domain.

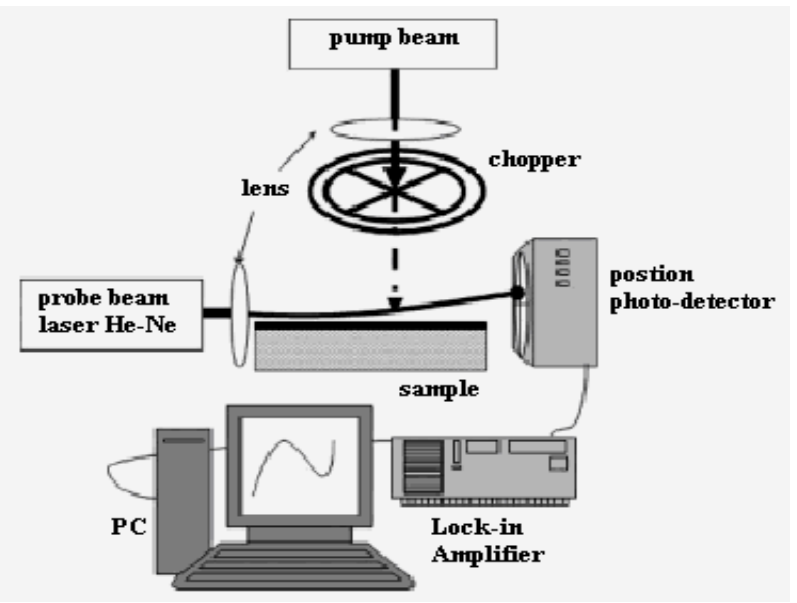

Figure 2. Experimental setup

\section{Results and Discussions}

In this work we have investigated Te-doped and non intentionally (n.i.d.) doped commercial GaSb bulk samples. The n.i.d. is usually $\mathrm{P}$ type semiconductor but the doped samples are of $\mathrm{N}$ type. Firstly, Hall effect measurements are used to determine the free carrier concentration given in table 1 .

Table 1. thermal diffusivity, non radiative lifetime and surface recombination velocity of $\mathrm{GaSb}$ samples

\begin{tabular}{|c|c|c|c|c|c|}
\hline $\begin{array}{c}\text { GaSb } \\
\text { samples }\end{array}$ & Dopant & $\begin{array}{c}\text { Free carrier } \\
\text { density } \\
(\mathrm{cm}-3)\end{array}$ & $\begin{array}{c}\text { Thermal } \\
\text { diffusivity } \\
\mathrm{Ds}(\mathrm{cm} 2 \\
\mathrm{s}-1)\end{array}$ & $\begin{array}{c}\text { Non } \\
\text { radiative } \\
\text { lifetime } \\
\mathrm{nr}(\mathrm{ns})\end{array}$ & $\begin{array}{c}\text { Surface re- } \\
\text { combination } \\
\text { velocity S } \\
\text { (cm s-1) }\end{array}$ \\
\hline 1 & n.i.d & 1.371017 & 0.235 & 1100 & 0.8105 \\
\hline 2 & $\mathrm{Te}$ & 5.11017 & 0.241 & 740 & 0.25105 \\
\hline 3 & $\mathrm{Te}$ & 9.621017 & 0.252 & 52 & 0.42105 \\
\hline 4 & $\mathrm{Te}$ & 2.31018 & 0.31 & 6 & 2.3105 \\
\hline
\end{tabular}

Then, photothermal deflection technique is carried out in order to measure the non radiative lifetime $\tau$ and the surface recombination velocity of the photogenerated minority carriers due to heating beam. In figures 3 and 4, are shown the experimental amplitude and phase curves for all the $\mathrm{GaSb}$ samples. These curves are compared to those obtained from the theoretical simulation using the above theoretical model. The best fitted theoretical curves are drawn on the same figures.
Theoretical simulations show that we can easily determine surface recombination velocity $S$ or non radiative lifetime $\tau$. However, the sensitivity of this model for bulk semiconductor is limited to the range of $1-10^{7} \mathrm{~cm} \mathrm{~s}^{-1}$ for $\mathrm{S}$ and $10^{-3}-$ $10^{-10} \mathrm{~s}$ for $\tau$.

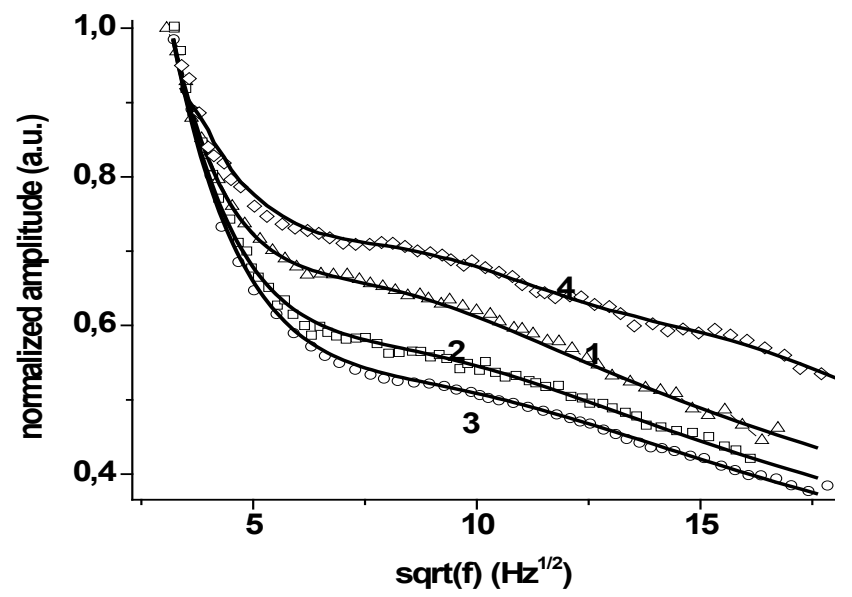

Figure 3. Amplitude of measured photothermal signal for undoped and $\mathrm{Te}$ doped $\mathrm{GaSb}$ samples (dots), fitted with theoretical amplitude (solid line)

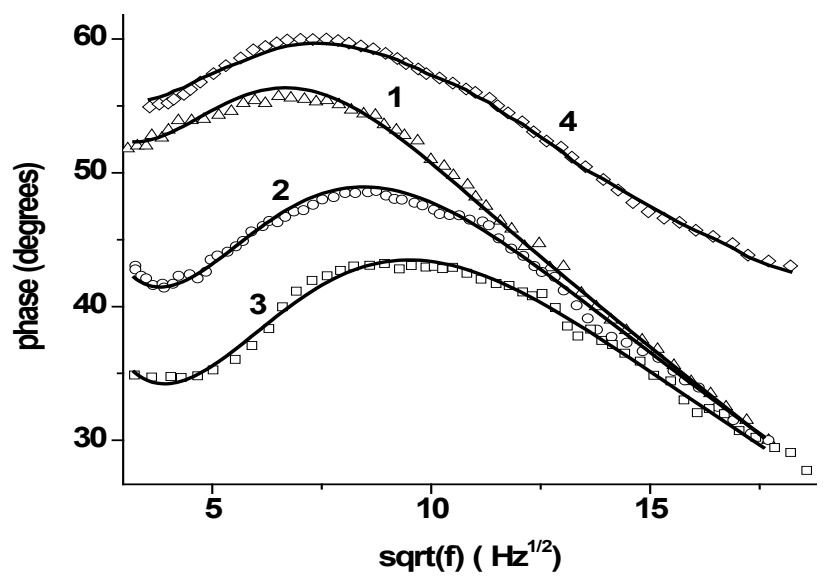

Figure 4. Phase of measured photothermal signal for undoped and Te doped GaSb samples (dots), fitted with theoretical phase (solid line)

The optimum values of and $\mathrm{S}$ obtained from the fitting of theoretical curves to experimental ones are reported on table 1. We can notice here, that non-radiative lifetime is enhanced by increasing the doping concentration. This feature is explained by the high amount of recombination centers introduced by doping. For Te doped samples, we notice that measured surface recombination velocity $\mathrm{S}$ (table I) increases with doping concentration. This is probably due to additional dangling bonds at the sample surface[14]. Although, $\mathrm{S}$ is greater for low Te doped GaSb (sample 2) than for undoped $\mathrm{GaSb}$ (sample 1), because the effective mass of the photoexcited carriers in p-type semiconductor (sample 1) is greater than in n- type one (sample 2)[15].

In figure 5 , we have drawn the non radiative lifetime versus $1 / \mathrm{N} 2$ where $\mathrm{N}$ is the doping concentration. This curve shows a linear behavior following the relation: $\tau=1 /\left(C N^{2}\right)$

The Auger recombination coefficient obtained from the linear fit is $\mathrm{C}=3.410-29 \mathrm{~cm} 6 . \mathrm{s}-1$. This value is in the range of those measured by other authors[16]. 


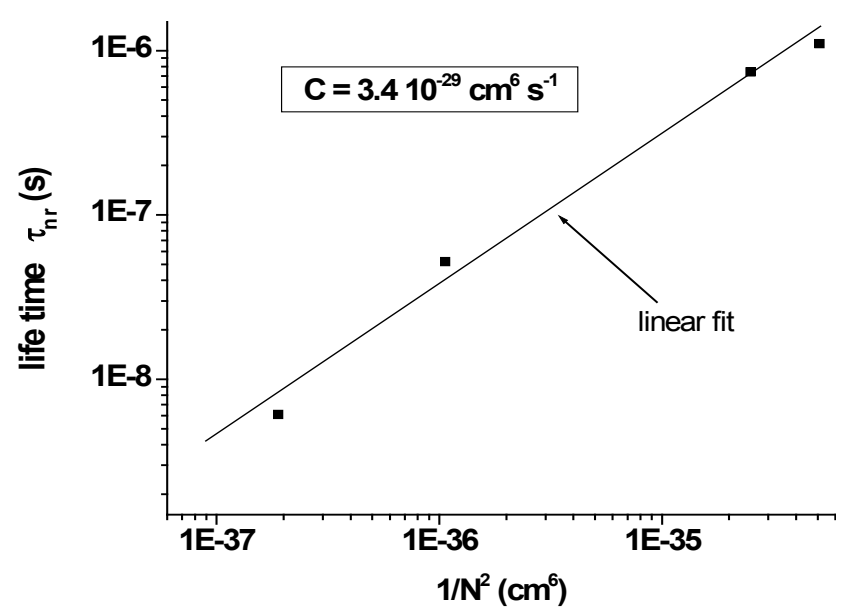

Figure 5. Non radiative lifetime $\tau_{\mathrm{nr}}$ of the photoexcited carriers versus $1 / \mathrm{N}^{2}$

The experimental phase curves (figure 4) show that while doping concentration is increased, a shift of the maximum toward higher frequencies is noticed. This shift is due to an improvement of the thermal diffusivity, which can be explained by tow factors: the increase of free carrier concentration and the enhancement of their mobility. All the obtained thermal diffusivity values are reported on table 1 . The curve of thermal diffusivity versus N2, shown on figure 6 , reveals a linear behavior with a slope of 1.34 10-38 cm8 s-1.

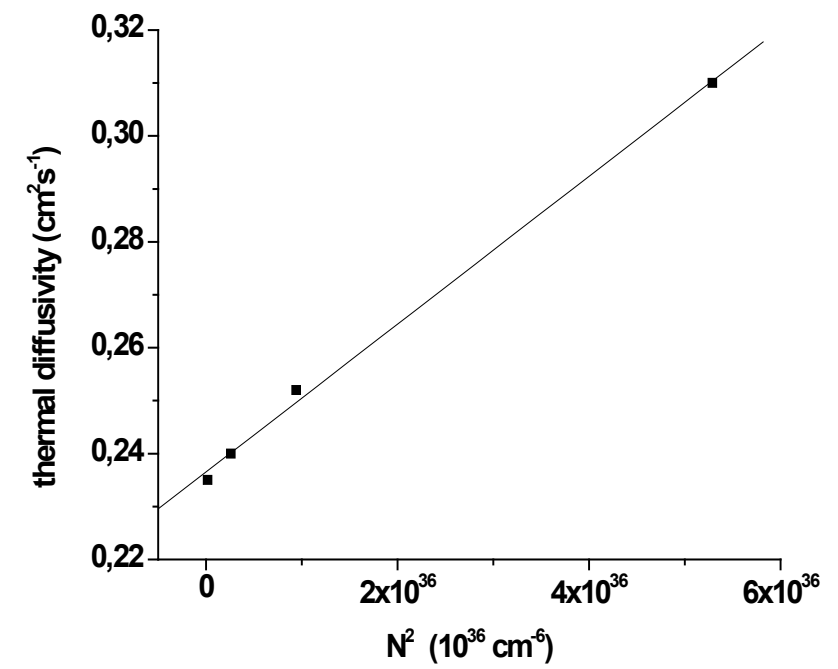

Figure 6. Thermal diffusivity obtained from photothermal signal versus $1 / \mathrm{N}^{2}(\mathrm{~N}$ is the carrier concentration)

Finally, from the comparison between figures 5 and 6 , we can conclude that thermal diffusivity of GaSb vary as the inverse of lifetime : $D_{s}=\alpha / \tau+\beta$; where $\alpha=0.410^{-9} \mathrm{~cm}^{2}$ and $\beta=0.236 \mathrm{~cm}^{2} \mathrm{~s}^{-1}$. This reveals a correlation between thermal and carrier diffusion processes which is probably due to the predominance of free carriers contribution to heat transport.

\section{Conclusions}

Photothermal deflection is used in order to determine surface recombination velocity and non-radiative life time for bulk GaSb semiconductor. For this purpose, the two components (phase and amplitude) of the measured photothermal signal are compared to the corresponding theoretical curves. The results show that while increasing of doping concentration, lifetime decreases and surface recombination velocity increases.

On the other hand we have revealed a correlation between non radiative lifetime and thermal diffusivity, this proves the predominance of free carriers contribution to both heat and electronic transport processes.

\section{REFERENCES}

[1] S. Anikeev, D. Doetsky, G. Belenky, and S. Luryi, C. A. Wang, J. M. Borrego, G. Nichols, "Measurement of the Auger recombination rate in p-type $0.54 \mathrm{eV} \mathrm{GaInAsSb}$ by time resolved photoluminescence", Appl. Phys. Lett. 83 (16), 3317-3319 (2003)

[2] Z. H. Chen, R. Bleiss, A. Mandelis, A. Buczkowski and F. Shimura,"Photothermal rate- window spectrometry for noncontact bulk lifetime measurements in semiconductors", J. Appl. Phys. 73 (10), 5043-5048 (1993)

[3] F. Saadallah, N. Yacoubi, F. Genty and C. Alibert, “ Investigation of thermal and optical properties of distributed Bragg reflectors by photothermal deflection spectroscopy" Appl. Opt. 41, 7561-7568 (2002)

[4] D. M. Todorovic, P. M. Nicolic, M. D. Dramicanin, D. G. Vasiljevic, and Z. D. Ristovski, "Photoacoustic frequency heat transmission technique: thermal and carrier transport parameters measurements in silicon", J. Appl. Phys. 78, 5750-5755 (1995)

[5] Jordi Sancho-Parramon, Josep Ferré-Borrull, Salvador Bosch, Anna Krasilnikova, and Jiri Bulir,'New calibration method for UV-VIS photothemal deflection spectroscopy set-up", Appl. Surface Science 253 (1), 158-162 (2006)

[6] A. N. Petrovsky, A. O. Salnick, V. V. Zuev, V. V. Grigoryev, and M. M. Mekhtiev, in Photothermal and Photoacoustic Phenomena III, edited by D. Bicanic springer series in Optical Sciences, vol.69,( Springer, Berlin, Heidelberg 1992) p. 390

[7] C. Khélia, K. Boubaker, T. Ben Nasrallah, M. Amlouk, S. Belgacem, F. Saadallah, N. Yacoubi,"Morfological and thermal properties of $\beta \mathrm{SnS}_{2}$ crystals grown by spray pyrolysis technique" J. Crystal Growth 311 (4), 1032-1035 (2009)

[8] F. Saadallah, N. Yacoubi, F. Genty and C. Alibert," Photothermal investigation of thermal and optical properties of GaAlAsSb and AlAsSb thin layers" J. Appl. Phys. 94 (8), 5041-5048 (2003)

[9] J. C. Murphy and L. C. Aamodt,"Photothermal Spectroscopy using Optical Beam Probing: Mirage Effect" J. Appl. Phys.51, 4580-4588 (1980)

[10] I. Delgadillo, M. Vargas, A. Cruz-Orea, J. J. Alvarado-Gil, R. Baquero, F. Sanchez-Sinencio, H. Varg, "Photoacoustic CdTe surface characterization”, Appl. Phys. B. 64, 97-101 (1997)

[11] E. Marın, I. Riech, P. Diaz, J. J. Alvarado-Gil, R. Baquero, J. 
G. Mendoza-Alvarez, H. Vargas, A. Cruz-Orea and M. Vargas, "Photoacoustic determination of non-radiative carrier lifetimes”, J. Appl. Phys. 83 (5), 2604-2609 (1998)

[12] Anita R. Warrier, Tina Sebastian, C. Sudha Kartha, and K. P. Vijayakumar, "Transverse photothermal beam deflection technique for determining the transport properties of semiconductor thin films", J. Appl. Phys. 107, 073701 (2010)

[13] A. Pinto Neto, H. Vargas, N.F. Leite and L.C.M. Miranda, "Photoacoustic investigation of semiconductors: influence of carrier diffusion and recombination in PbTe and Si", Phys. Rev.B. 40 (6), 9971-9979 (1989)
[14] I. Reich, P. Diaz, and E. Marian, "Study of non radiative recombination mechanisms in semiconductors by photoacoustic measurements", Phys. Stat. Sol. (b) 220, 305-308 (2000)

[15] Sajan. D. George, Dilna. S. P. Radhakrishnan, C. P. G. Vallabhan, and V. P. N. Nampoori, "Photoacoustic measurement of transport properties in doped GaAs epitaxial layers", Phys. Stat. Sol. (a) 195 (2), 416-421 (2003)

[16] A. Joullié, "New developments in mid-infrared Sb-based lasers", J. Phys. France 9 (1999), Pr2-79 - Pr2-95 